\author{
Milan S. Cajić \\ PhD Student \\ University of Belgrade \\ Faculty of Mechanical Engineering \\ Research Assistan \\ Mathematical Institute of the SASA (Serbian \\ academy of Sciences and Arts) \\ Mihailo P. Lazarević \\ Full Professor \\ University of Belgrade \\ Faculty of Mechanical Engineering
}

\section{Determination of Joint Reactions in a Rigid Multibody System, Two Different Approaches}

In this paper two different methods for determination of frictionless joint reaction forces and moments are presented. The considered multibody system has an open kinematic chain structure. The first method refers to the determination of resultant joint reaction forces and moments based on the Rodrigues approach suitable for computation in a symbolic form. The second method presented is the method based on the so-called vectors of the body mass moments and vector rotators coupled for a pole and oriented axes. Both approaches are presented and discussed on the threelike rigid multibody system.

Keywords: multibody dynamics, resultant joint reaction forces and moments, vectors of body mass moments, vector rotators

\section{INTRODUCTION}

Determination of joint reactions is of great importance in dynamics of multibody systems, machines and mechanisms. In this paper, the Coulomb friction forces are not considered but it is necessary if we want to get a more realistic picture of the dynamics of a multibody system (see e.g. [1-4]).

In the recently published paper [5] the broad overview of research papers that deals with the determination of joint reactions have been presented. The papers based on Newton-Euler formalism with recursive and non-recursive kinematic and dynamic relations are cited there. The main part of that paper is determination of joint reactions based on fictitious bodies method and general method based on Lagrange's equations. Therefore, for better understanding of this problem the reader is referred to the [5] and [6].

In [8-12] a different approach to the problem of determination of joint reactions and kinetic pressures is used. By introducing the vector method based on the vectors of the body mass moments [8, 9] and vector rotators coupled for pole and oriented axes it is possible to obtain vector expressions for determination of joint reactions and kinetic pressures for a rigid body dynamics.

In this paper, both methods are illustrated on the threelike rigid multibody system. Expressions for the linear and angular momentum as well as expressions for their derivatives are derived. The procedure for determination of the resultant joint reaction forces and moments is presented and some comments on both methods are given.

\section{DETERMINATION OF JOINT REACTIONS USING RODRIGUES APPROACH}

In this section, formulation of a general method for

Received: June 2014, Accepted: May 2015

Correspondence to: Mihailo P. Lazarević

Faculty of Mechanical Engineering,

Kraljice Marije 16, 11120 Belgrade 35, Serbia

E-mail: mlazarevic@mas.bg.ac.rs

doi:10.5937/fmet1602165C

() Faculty of Mechanical Engineering, Belgrade. All rights reserved determination of the resultant joint reaction forces and moments is given. If the robotic system has the form of a multibody system with open kinematic chain structure , then we can denote the first and the fixed base body with $\left(V_{l}\right)$ and the following bodies in the system we denote with subscripts incremented for one with regard to the subscript of its preceding adjacent body $\left(V_{2}, \ldots . V_{k}, \ldots V_{i}, V_{i+1}, V_{i+2}, \ldots, V_{n}\right)$. Also, the geometry of the system has been defined by unit vectors $\vec{e}_{i}, i=1,2, \ldots, j, . ., n$ where unit vectors $\vec{e}_{i}$ describe the axis of rotation (translation) of the $i$-th segment with respect to the previous segment and as well as vectors $\vec{\rho}_{i}$ and $\vec{\rho}_{i i}$, (see $\left.[6,7]\right)$. In a general case, the configuration of the multibody system can be defined by the vector of joint (internal) generalized coordinates $q$ of the dimension $n,(q)=\left(q^{1}, q^{2}, \ldots, q^{n}\right)$ where $q$ are the relative angles of rotation (in the case of revolute joints) and the relative displacements (in the case of prismatic joints). If all the variables $q^{i}, i=1,2, \ldots, n$ are zero, it is said that the robotic system is in the reference configuration (position).

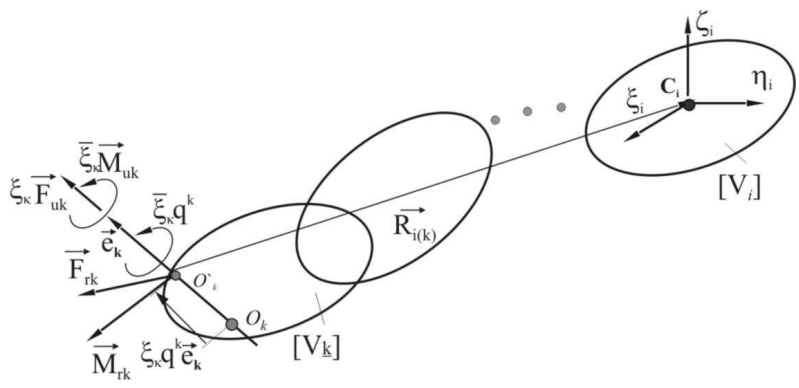

Figure 1. Rigid multibody system with the open kinematic

$$
\text { chain structure }\left(\left[V_{k}\right] \rightarrow\left[V_{j}\right]\right)
$$

To determine the resultant joint reaction force and the moment in some $k$-th joint let us first consider the equation for linear momentum of all segments of a rigid multibody system (see Figure 1) behind the $k$-th segment as: 


$$
\vec{K}=\sum_{i: k(\leq i)} \vec{K}_{i}=\sum_{i} m_{i} \vec{v}_{C i}
$$

where $\vec{v}_{C i}$ and $m_{i}$ denote the velocity of the mass center and the mass of the body $\left(V_{i}\right)$. After diferentiation of (1) we obtain:

$$
\frac{d \vec{K}}{d t}=\sum_{i} m_{i} \vec{a}_{C i}=\vec{F}_{R(r v)}+\sum_{i} m_{i} \vec{g}+\xi_{k} \vec{F}_{u k}
$$

From (2) we can obtain a formula for determining of the resultant joint reaction force $\vec{F}_{R(r v)}$ in the $k$-th joint as:

$$
\vec{F}_{R(r v)}=\sum_{i} m_{i}\left(\vec{a}_{C i}-\vec{g}\right)-\xi_{k} F_{u k} \vec{e}_{k}
$$

where $\vec{a}_{C i}$ and $\vec{g}$ are acceleration of mass center and acceleration of gravity, respectively. Also, in (3) $F_{u k}$ is an actuator force, $\vec{e}_{k}$ denotes unit vector describing the axis of rotation (translation) of the $k$-th segment with respect to the previous segment, whereas parameter $\xi_{k}$ deter-mines the type of the $k$-th joint. The parameter $\xi_{k}=0$ if the considered joint is revolute and $\xi_{k}=1$ in case of a prismatic joint, where $\bar{\xi}_{k}=1-\xi_{k}$ is also introduced. The velocity $\vec{v}_{C i}$ and acceleration $\vec{a}_{C i}$ of mass center of $i$-th segment can be evaluated from the following expressions:

$$
\begin{aligned}
& \vec{v}_{C i}=\sum_{\alpha=1}^{i} \vec{T}_{\alpha(i)} \dot{q}^{\alpha}, \\
& \vec{a}_{C i}=\sum_{\alpha=1}^{i} \vec{T}_{\alpha(i)} \ddot{q}^{\alpha}+\sum_{\alpha=1}^{i} \sum_{\beta=1}^{i} \vec{\Gamma}_{\alpha \beta(i)} \dot{q}^{\alpha} \dot{q}^{\beta}
\end{aligned}
$$

where $\dot{q}^{\alpha}, \dot{q}^{\beta}$ are generalized velocities, $\vec{T}_{i(\alpha)}$ are quasibase vectors (for details see [6]) and vectors $\vec{\Gamma}_{\alpha \beta(i)}$ are in the following form:

$$
\begin{array}{ll}
\vec{\Gamma}_{\alpha \beta(i)}=\bar{\xi}_{\alpha} \vec{e}_{\alpha} \times \vec{T}_{\beta(i)}, \quad \forall \alpha \leq \beta, \\
\vec{\Gamma}_{\alpha \beta(i)}=\bar{\xi}_{\alpha} \vec{e}_{\beta} \times \vec{T}_{\alpha(i)}, & \forall \alpha>\beta, \\
\vec{\Gamma}_{\alpha \beta(i)}=0, & \forall \alpha>i
\end{array}
$$

Resultant joint reaction force $\vec{F}_{R(r v)}$ with respect to inertial fixed reference frame $O x y z$ (null base) can be calculated as:

$$
\begin{aligned}
& \left\{\vec{F}_{R(r v)}^{(0)}\right\}=\sum_{i} m_{i}\left(\vec{a}_{C i}^{(0)}-\left[A_{0, i}\right]\{\vec{g}\}\right) \\
& -\xi_{k} F_{u k}\left[A_{0, k}\right]\left\{\vec{e}_{k}\right\},
\end{aligned}
$$

where the appropriate Rodrigues matrices of transformation are:

$$
\begin{aligned}
& {\left[A_{j-1, j}\right]=[I]+\left[e_{j}^{d(j)}\right]^{2}\left(1-\cos \left(q^{j}\right)\right)} \\
& +\left[e_{j}^{d(j)}\right] \sin \left(q^{j}\right),
\end{aligned}
$$

as well as square matrix $\left[e_{j}^{d(j)}\right]$ represents dual object of vector:

$$
\begin{aligned}
& \left(\vec{e}_{j}^{(j)}\right)=\left(e_{\xi j}, e_{\eta_{j}}, e_{\zeta j}\right), \\
& {\left[e_{j}^{d(j)}\right]=\left[\begin{array}{ccc}
0 & -e_{\zeta j} & e_{\eta j} \\
e_{\zeta j} & 0 & -e_{\xi_{j}} \\
-e_{\eta j} & e_{\xi_{j}} & 0
\end{array}\right]}
\end{aligned}
$$

In a similar maner, the velocity and acceleration of the center of mass with respect to the null base are:

$$
\begin{aligned}
& \left\{\vec{v}_{C i}^{(0)}\right\}=\sum_{\alpha=1}^{i}\left\{\vec{T}_{\alpha(i)}^{(0)}\right\} \dot{q}^{\alpha}, \\
& \left\{\vec{a}_{C i}^{(0)}\right\}=\sum_{\alpha=1}^{i}\left\{\vec{T}_{\alpha(i)}^{(0)}\right\} \ddot{q}^{\alpha}+\sum_{\alpha=1}^{i} \sum_{\beta=1}^{i}\left\{\vec{\Gamma}_{\alpha \beta(i)}^{(0)}\right\} \dot{q}^{\alpha} \dot{q}^{\beta}
\end{aligned}
$$

and vectors $\vec{\Gamma}_{\alpha \beta(i)}$ with respect to the null base are

$$
\begin{aligned}
& a=\underline{\alpha, \beta}, \quad b=\overline{\alpha, \beta}, \\
& \left\{\vec{\Gamma}_{\alpha \beta(i)}^{(0)}\right\}=\bar{\xi}_{a} \vec{e}_{a} \times \vec{T}_{b(i)}^{(b)}
\end{aligned}
$$

where quasibase vectors $\vec{T}_{b(i)}^{(b)}$ can be obtained in the following form

$$
\begin{aligned}
& \vec{T}_{b(i)}^{(b)}=\bar{\xi}_{b}\left[e_{b}^{d}\right]\left\{\sum _ { k = b } ^ { i } [ A _ { b , k } ] \left(\left\{\vec{\rho}_{k k}\right\}+\xi_{k} q^{k}\left\{\vec{e}_{k}\right\}\right.\right. \\
& \left.\left.+\left[A_{b, i}\right]\left\{\vec{\rho}_{i}\right\}\right)\right\}+\xi_{b}\left\{\vec{e}_{b}\right\},
\end{aligned}
$$

Further, angular momentum of $i$-th segment with respect to the pole in $k$-th joint of a multibody system is given in the form:

$$
\vec{L}_{O K(i)}=\vec{L}_{C i}+\overrightarrow{O_{k}^{\prime} C_{i}} \times m_{i} \vec{v}_{C i}
$$

Total angular momentum of all segments behind the $k$-th joint is:

$$
\vec{L}_{O K}=\sum_{i}\left(\vec{L}_{C i}+\overrightarrow{O_{k}^{\prime} C_{i}} \times m_{i} \vec{v}_{C i}\right)
$$

After differentiation of the last expression, we obtain

$$
\begin{aligned}
& \frac{d \vec{L}_{O K}}{d t}=\sum_{i} \frac{d \vec{L}_{C i}}{d t}+\frac{d \overrightarrow{O_{k}^{\prime} C_{i}}}{d t} \times m_{i} \vec{v}_{C i} \\
& +\overrightarrow{O_{k}^{\prime} C_{i}} \times m_{i} \vec{a}_{C i},
\end{aligned}
$$

and

$$
\frac{d \overrightarrow{O_{k}^{\prime} C_{i}}}{d t}=\vec{v}_{C i}-\vec{v}_{O k}, \vec{R}_{i(k)}=\overrightarrow{O_{k}^{\prime} C_{i}}
$$

where $\vec{L}_{C i}$ is angular momentum with respect to the mass centre of the $i$-th body, we can present (14) in the following form 


$$
\frac{d \vec{L}_{O K}}{d t}=\sum_{i}\left(\frac{d \vec{L}_{C i}}{d t}-\vec{v}_{O k} \times m_{i} \vec{v}_{C i}+\vec{R}_{i(k)} \times m_{i} \vec{a}_{C i}\right)
$$

Resultant reaction moment $\vec{M}_{O k R}$ of all forces acting on segments behind $k$-th joint is:

$$
\vec{M}_{O k R}=\frac{d \vec{L}_{O k}}{d t}+\vec{v}_{O k} \times \sum_{i} m_{i} \vec{v}_{C i}
$$

Equations (14) and (15) yields :

$$
\begin{aligned}
& \vec{M}_{O k(r v)}=\sum_{i}\left(\frac{d \vec{L}_{C i}}{d t}+\vec{R}_{i(k)} \times m_{i}\left(\vec{a}_{C i}-\vec{g}\right)\right) \\
& -\bar{\xi}_{k} M_{u k} \vec{e}_{k} .
\end{aligned}
$$

When we take into account the actuator torque $M_{u k}$ and torque due to the gravitational forces $m_{i} \vec{g}$ we have the final form of the resultant joint reaction moment in the $k$-th joint as

$$
\begin{aligned}
& \vec{M}_{O k(r v)}=\sum_{i}\left(\frac{d \vec{L}_{C i}}{d t}+\vec{R}_{i(k)} \times m_{i}\left(\vec{a}_{C i}-\vec{g}\right)\right) \\
& -\bar{\xi}_{k} M_{u k} \vec{e}_{k} .
\end{aligned}
$$

On the other side, first derivative $d \vec{L}_{C i} / d t$ can be obtained in the final form, [6] as:

$$
\frac{d \vec{L}_{C i}}{d t}=\left[\omega_{i}^{d}\right]\left[J_{C i}\right]\left\{\omega_{i}\right\}+\left[J_{C i}\right]\left\{\varepsilon_{i}\right\}
$$

where $\omega_{i}, \varepsilon_{i}$ and $J_{C i}$ are:

1) angular velocity of the $i$-th body in vector form

$$
\vec{\omega}_{i}=\sum_{\alpha=1}^{i} \bar{\xi}_{\alpha} \vec{e}_{\alpha} \dot{q}^{\alpha}, \quad \alpha \leq i
$$

as well as in a matrix form

$$
\left\{\omega_{i}\right\}=\left\{\begin{array}{l}
\omega_{i \xi} \\
\omega_{i \eta} \\
\omega_{i \zeta}
\end{array}\right\}, \quad\left[\omega_{i}^{d}\right]=\left[\begin{array}{ccc}
0 & -\omega_{i \zeta} & \omega_{i \eta} \\
\omega_{i \zeta} & 0 & -\omega_{i \xi} \\
-\omega_{i \eta} & \omega_{i \xi} & 0
\end{array}\right] \text { (22) }
$$

2) angular acceleration of the $i$-th body is

$$
\begin{aligned}
& \vec{\varepsilon}_{i}=\sum_{\alpha=1}^{i} \bar{\xi}_{\alpha} \vec{e}_{\alpha} \ddot{q}^{\alpha}+\sum_{\alpha=1}^{i} \sum_{\beta=1}^{\alpha} \bar{\xi}_{\alpha} \bar{\xi}_{\beta} \vec{e}_{\beta} \times \vec{e}_{\alpha} \dot{q}^{\alpha} \dot{q}^{\beta} \\
& \alpha \leq i, \quad \beta \leq \alpha
\end{aligned}
$$

3) mass inertia tensor $J_{C i}$ of the $i$-th body is

$$
\begin{aligned}
& {\left[J_{C i}\right]^{\text {def }}=-\int_{(V)}\left[\tau^{d}\right]^{2} d m=\int_{(V)}\left[\begin{array}{ccc}
\tau_{\eta}^{2}+\tau_{\zeta}^{2} & -\tau_{\xi} \tau_{\eta} & -\tau_{\xi} \tau_{\zeta} \\
-\tau_{\eta} \tau_{\xi} & \tau_{\zeta}^{2}+\tau_{\xi}^{2} & -\tau_{\eta} \tau_{\zeta} \\
-\tau_{\zeta} \tau_{\xi} & -\tau_{\zeta} \tau_{\eta} & \tau_{\xi}^{2}+\tau_{\eta}^{2}
\end{array}\right] d m} \\
& {\left[J_{C i}\right]=\left[\begin{array}{ccc}
J_{\zeta} & -J_{\xi \eta} & -J_{\xi \zeta} \\
-J_{\eta \xi} & J_{\eta} & -J_{\eta \zeta} \\
-J_{\zeta \xi} & -J_{\zeta \eta} & J_{\xi}
\end{array}\right]}
\end{aligned}
$$

where $\vec{\tau}$ is the position vector with respect to the body mass centre

$$
\{\vec{\tau}\}=\left\{\begin{array}{c}
\tau_{\xi} \\
\tau_{\eta} \\
\tau_{\zeta}
\end{array}\right\}, \quad\left[\tau^{d}\right]=\left[\begin{array}{ccc}
0 & -\tau_{\zeta} & \tau_{\eta} \\
\tau_{\zeta} & 0 & -\tau_{\xi} \\
-\tau_{\eta} & \tau_{\xi} & 0
\end{array}\right]
$$

For more details, the reader is reffered to [6] and [7].

\subsection{Determination of joint reaction forces and moments for the three-like rigid multibody system}

When we consider the simple three rigid body system, (Fig. 2) where the linear momentum of all bodies in the system is:

$$
\vec{K}=\sum_{i=1}^{3} \vec{K}_{i}=\sum_{i=1}^{3} m_{i} \vec{v}_{C i}
$$

where velocities of body mass centers with respect to the local inertial coordinate systems are

$$
\begin{aligned}
& \vec{v}_{C 1}=\vec{T}_{(1) 1} \dot{q}^{1} ; \vec{v}_{C 2}=\vec{T}_{(1) 2} \dot{q}^{1}+\vec{T}_{(2) 2} \dot{q}^{2} ; \\
& \vec{v}_{C 3}=\vec{T}_{(1) 3} \dot{q}^{1}+\vec{T}_{(2) 3} \dot{q}^{2}+\vec{T}_{(3) 3} \dot{q}^{3}
\end{aligned}
$$

and quaibase vectors

$$
\begin{aligned}
& \vec{T}_{(1) 1}=\bar{\xi}_{1} \vec{e}_{1} \times\left[\vec{\rho}_{11}+\vec{\rho}_{1}\right]+\xi_{1} \vec{e}_{1}, \\
& \vec{T}_{(1) 2}=\bar{\xi}_{1} \vec{e}_{1} \times\left[\vec{\rho}_{11}+\vec{\rho}_{22}+\vec{\rho}_{2}\right]+\xi_{1} \vec{e}_{1}, \\
& \vec{T}_{(1) 3}=\bar{\xi}_{1} \vec{e}_{1} \times\left[\vec{\rho}_{11}+\vec{\rho}_{22}+\vec{\rho}_{33}+\vec{\rho}_{3}\right]+\xi_{1} \vec{e}_{1}, \\
& \vec{T}_{(2) 2}=\bar{\xi}_{2} \vec{e}_{2} \times\left[\vec{\rho}_{22}+\vec{\rho}_{2}\right]+\xi_{2} \vec{e}_{2}, \\
& \vec{T}_{(2) 3}=\bar{\xi}_{2} \vec{e}_{2} \times\left[\vec{\rho}_{22}+\vec{\rho}_{33}+\vec{\rho}_{3}\right]+\xi_{2} \vec{e}_{2}, \\
& \vec{T}_{(3) 3}=\bar{\xi}_{3} \vec{e}_{3} \times\left[\vec{\rho}_{33}+\vec{\rho}_{3}\right]+\xi_{3} \vec{e}_{3},
\end{aligned}
$$

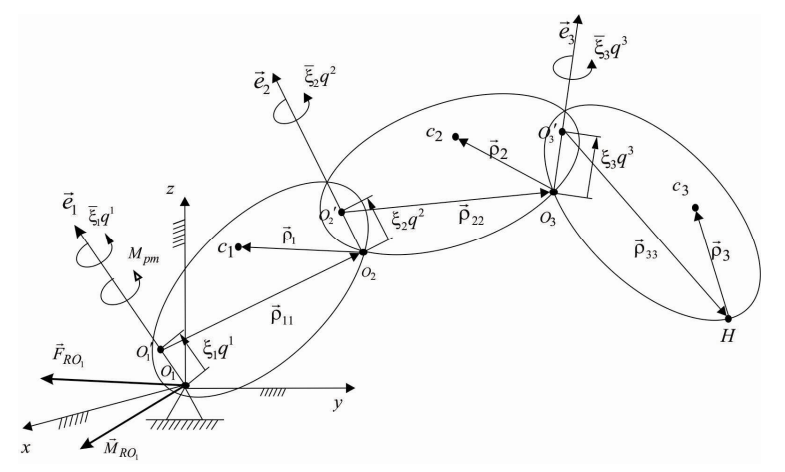

Figure 2. Three-like rigid multibody system with vectors using Rodrigues approach

After derivation of a linear momentum by the following formula:

$$
\frac{d \vec{K}}{d t}=\sum_{i=1}^{3} m_{i} \vec{a}_{C i}=\vec{F}_{R(r v)}+\sum_{i=1}^{3} m_{i} \vec{g}
$$

we can easily find the resultant joint reaction force for the first and fixed joint of the three body system as

$$
\vec{F}_{R(r v)}=\sum_{i=1}^{3} m_{i}\left(\vec{a}_{C i}-\vec{g}_{i}\right)-\xi_{k} F_{u k} \vec{e}_{k}
$$

in case of revolute joint we have (30) without the last term in the form: 


$$
\begin{aligned}
& \vec{F}_{R(r v)}=m_{1}\left(\vec{a}_{C 1}-\vec{g}_{1}\right)+ \\
& +m_{2}\left(\vec{a}_{C 2}-\vec{g}_{2}\right)+m_{3}\left(\vec{a}_{C 3}-\vec{g}_{3}\right)
\end{aligned}
$$

Accelerations of body mass centers are

$$
\begin{aligned}
& \vec{a}_{C 1}=\vec{T}_{1(1)} \ddot{q}^{1}+\vec{\Gamma}_{11(1)} \dot{q}^{1} \dot{q}^{1} \\
& \vec{a}_{C 2}=\vec{T}_{2(1)} \ddot{q}^{1}+\vec{T}_{2(2)} \ddot{q}^{2}+\vec{\Gamma}_{11(2)} \dot{q}^{1} \dot{q}^{1}+\vec{\Gamma}_{12(2)} \dot{q}^{1} \dot{q}^{2} \\
& +\vec{\Gamma}_{21(2)} \dot{q}^{2} \dot{q}^{1}+\vec{\Gamma}_{22(2)} \dot{q}^{2} \dot{q}^{2}, \\
& \vec{a}_{C 3}=\vec{T}_{3(1)} \ddot{q}^{1}+\vec{T}_{3(2)} \ddot{q}^{2}+\vec{T}_{3(3)} \ddot{q}^{3}+\vec{\Gamma}_{11(3)} \dot{q}^{1} \dot{q}^{1}+ \\
& +\vec{\Gamma}_{12(3)} \dot{q}^{1} \dot{q}^{2}+\vec{\Gamma}_{13(3)} \dot{q}^{1} \dot{q}^{3}+\vec{\Gamma}_{21(3)} \dot{q}^{2} \dot{q}^{1}+ \\
& +\vec{\Gamma}_{22(3)} \dot{q}^{2} \dot{q}^{2}+\vec{\Gamma}_{23(3)} \dot{q}^{2} \dot{q}^{3}++\vec{\Gamma}_{31(3)} \dot{q}^{3} \dot{q}^{1}, \\
& +\vec{\Gamma}_{32(3)} \dot{q}^{3} \dot{q}^{2}+\vec{\Gamma}_{33(3)} \dot{q}^{3} \dot{q}^{3}
\end{aligned}
$$

and $\vec{\Gamma}_{\alpha \beta(i)}$ vectors are

$$
\begin{aligned}
& \vec{\Gamma}_{11(1)}=\bar{\xi}_{1} \vec{e}_{1} \times \vec{T}_{1(1)}, \vec{\Gamma}_{11(2)}=\bar{\xi}_{1} \vec{e}_{1} \times \vec{T}_{1(2)} \\
& \vec{\Gamma}_{12(2)}=\bar{\xi}_{1} \vec{e}_{1} \times \vec{T}_{2(2)}=\vec{\Gamma}_{21(2)} \\
& \vec{\Gamma}_{21(2)}=\bar{\xi}_{1} \vec{e}_{1} \times \vec{T}_{2(2)}, \vec{\Gamma}_{22(2)}=\bar{\xi}_{2} \vec{e}_{2} \times \vec{T}_{2(2)} \\
& \vec{\Gamma}_{11(3)}=\bar{\xi}_{1} \vec{e}_{1} \times \vec{T}_{1(3)}, \vec{\Gamma} \sqrt{a^{2}+b^{2}}{ }_{12(3)}= \\
& =\bar{\xi}_{1} \vec{e}_{1} \times \vec{T}_{2(3)}=\vec{\Gamma}_{21(3)} \\
& \vec{\Gamma}_{13(3)}=\bar{\xi}_{1} \vec{e}_{1} \times \vec{T}_{3(3)}=\vec{\Gamma}_{31(3)}, \vec{\Gamma}_{21(3)}=\bar{\xi}_{1} \vec{e}_{1} \times \vec{T}_{2(3)} \\
& \vec{\Gamma}_{22(3)}=\bar{\xi}_{2} \vec{e}_{2} \times \vec{T}_{2(3)}, \vec{\Gamma}_{23(3)}=\bar{\xi}_{2} \vec{e}_{2} \times \vec{T}_{2(3)} \\
& \vec{\Gamma}_{31(3)}=\bar{\xi}_{1} \vec{e}_{1} \times \vec{T}_{3(3)}, \vec{\Gamma}_{32(3)}=\bar{\xi}_{2} \vec{e}_{2} \times \vec{T}_{3(3)}=\vec{\Gamma}_{23(3)} \\
& \vec{\Gamma}_{33(3)}=\bar{\xi}_{3} \vec{e}_{3} \times \vec{T}_{3(3)}
\end{aligned}
$$

Finaly, after using the (32) and (33) we obtain:

$$
\begin{aligned}
& \vec{F}_{R(r v)}=m_{1}\left(\vec{T}_{1(1)} \ddot{q}^{1}+\vec{\Gamma}_{11(1)}\left(\dot{q}^{1}\right)^{2}-\vec{g}_{1}\right) \\
& +m_{2}\left(\vec{T}_{2(1)} \ddot{q}^{1}+\vec{T}_{2(2)} \ddot{q}^{2}+\vec{\Gamma}_{11(2)}\left(\dot{q}^{1}\right)^{2}\right. \\
& \left.+2 \vec{\Gamma}_{12(2)} \dot{q}^{1} \dot{q}^{2}+\vec{\Gamma}_{22(2)}\left(\dot{q}^{2}\right)^{2}-\vec{g}_{2}\right) \\
& +m_{3}\left(\vec{T}_{3(1)} \ddot{q}^{1}+\vec{T}_{3(2)} \ddot{q}^{2}+\vec{T}_{3(3)} \ddot{q}^{3}+\right. \\
& +\vec{\Gamma}_{11(3)}\left(\dot{q}^{1}\right)^{2}+2 \vec{\Gamma}_{12(3)} \dot{q}^{1} \dot{q}^{2}+2 \vec{\Gamma}_{13(3)} \dot{q}^{1} \dot{q}^{3} \\
& \left.+\vec{\Gamma}_{22(3)}\left(\dot{q}^{2}\right)^{2}+2 \vec{\Gamma}_{23(3)} \dot{q}^{2} \dot{q}^{3}+\vec{\Gamma}_{33(3)}\left(\dot{q}^{3}\right)^{2}-\vec{g}_{3}\right) .
\end{aligned}
$$

Total angular momentum of a three body sytem with a pole in the first joint is:

$$
\begin{aligned}
& \vec{L}_{O K}=\sum_{i=1}^{3}\left(\vec{L}_{C i}+\overrightarrow{O_{k}^{\prime} C_{i}} \times m_{i} \vec{v}_{C i}\right)=\vec{L}_{C 1}+\vec{R}_{1(k)} \times m_{1} \vec{v}_{C 1} \\
& +\vec{L}_{C 2}+\vec{R}_{2(k)} \times m_{2} \vec{v}_{C 2}+\vec{L}_{C 3}+\vec{R}_{3(k)} \times m_{3} \vec{v}_{C 3} .
\end{aligned}
$$

Derivative of angular momentum is given by:

$\frac{d \vec{L}_{O K}}{d t}=\sum_{i=1}^{3}\left(\frac{d \vec{L}_{C i}}{d t}-\vec{v}_{O k} \times m_{i} \vec{v}_{C i}+\vec{R}_{i(k)} \times m_{i} \vec{a}_{C i}\right)$

In case the first joint is revolute, then for the resultant joint reaction moment we have

$$
\begin{aligned}
& \vec{M}_{O k(r v)}=\left(\frac{d \vec{L}_{C 1}}{d t}+\vec{R}_{1(k)} \times m_{1}\left(\vec{a}_{C 1}-\vec{g}\right)\right) \\
& +\left(\frac{d \vec{L}_{C 2}}{d t}+\vec{R}_{2(k)} \times m_{2}\left(\vec{a}_{C 2}-\vec{g}\right)\right) \\
& +\left(\frac{d \vec{L}_{C 3}}{d t}+\vec{R}_{3(k)} \times m_{3}\left(\vec{a}_{C 3}-\vec{g}\right)\right)-\bar{\xi}_{k} M_{u k} \vec{e}_{k},
\end{aligned}
$$

where $d \vec{L}_{C i} / d t$ for the three body system can be dermined as

$$
\begin{aligned}
& \frac{d \vec{L}_{C 1}}{d t}=\left[\omega_{1}^{d}\right]\left[J_{C 1}\right]\left\{\omega_{1}\right\}+\left[J_{C 1}\right]\left\{\varepsilon_{1}\right\}, \\
& \frac{d \vec{L}_{C 2}}{d t}=\left[\omega_{2}^{d}\right]\left[J_{C 2}\right]\left\{\omega_{2}\right\}+\left[J_{C 2}\right]\left\{\varepsilon_{2}\right\}, \\
& \frac{d \vec{L}_{C 3}}{d t}=\left[\omega_{3}^{d}\right]\left[J_{C 3}\right]\left\{\omega_{3}\right\}+\left[J_{C 3}\right]\left\{\varepsilon_{3}\right\}
\end{aligned}
$$

where $\omega_{i}$ and $\omega_{i}^{d}$ for $i=1,2,3$ are determined by using the (21) and (22) and $\varepsilon_{i}$ are

$$
\begin{aligned}
& \vec{\varepsilon}_{1}=\bar{\xi}_{1} \vec{e}_{1} \ddot{q}^{1}+\bar{\xi}_{1} \bar{\xi}_{1} \vec{e}_{1} \times \vec{e}_{1} \dot{q}^{1} \dot{q}^{1}=\bar{\xi}_{1} \vec{e}_{1} \ddot{q}^{1} \\
& \vec{\varepsilon}_{2}=\bar{\xi}_{1} \vec{e}_{1} \ddot{q}^{1}+\bar{\xi}_{2} \vec{e}_{2} \ddot{q}^{2}+\bar{\xi}_{1} \bar{\xi}_{1} \vec{e}_{1} \times \vec{e}_{1} \dot{q}^{1} \dot{q}^{1} \\
& +\bar{\xi}_{2} \bar{\xi}_{1} \vec{e}_{1} \times \vec{e}_{2} \dot{q}^{2} \dot{q}^{1}+\bar{\xi}_{2} \bar{\xi}_{2} \vec{e}_{2} \times \vec{e}_{2} \dot{q}^{2} \dot{q}^{2} \\
& \vec{\varepsilon}_{3}=\bar{\xi}_{1} \vec{e}_{1} \ddot{q}^{1}+\bar{\xi}_{2} \vec{e}_{2} \ddot{q}^{2}+\bar{\xi}_{3} \vec{e}_{3} \ddot{q}^{3}+\bar{\xi}_{1} \bar{\xi}_{1} \vec{e}_{1} \times \vec{e}_{1} \dot{q}^{1} \dot{q}^{1} \\
& +\bar{\xi}_{2} \bar{\xi}_{1} \vec{e}_{1} \times \vec{e}_{2} \dot{q}^{2} \dot{q}^{1}+\bar{\xi}_{2} \bar{\xi}_{2} \vec{e}_{2} \times \vec{e}_{2} \dot{q}^{2} \dot{q}^{2} \\
& +\bar{\xi}_{3} \bar{\xi}_{1} \vec{e}_{1} \times \vec{e}_{3} \dot{q}^{3} \dot{q}^{1}+\bar{\xi}_{3} \bar{\xi}_{2} \vec{e}_{2} \times \vec{e}_{3} \dot{q}^{3} \dot{q}^{2} \\
& +\bar{\xi}_{3} \bar{\xi}_{3} \vec{e}_{3} \times \vec{e}_{3} \dot{q}^{3} \dot{q}^{3}
\end{aligned}
$$

Even if expressions for determination of joint reactions are given here explicitly, it is possible to use some of the software for symbolic computation (e.g. Wolfram Mathematica), or to make computation algorithm using some of the programming languages (e.g. see [6]), and to determine in an easy way joint reactions for arbitrary number of rigid bodies in a system.

\section{DETERMINATION OF JOINT REACTIONS BY APPLYING THE METHOD BASED ON VECTORS OF BODY MASS MOMENTS AND VECTOR ROTATORS}

This method was first introduced in [8] where vectors of body mass moments were introduced by definitions. In monograph [9], the more extensive study of this method and nonlinear dynamics analysis of the heavy rotors and gyro-rotors have been carried out. This method was applied in [10-12] for vector analysis properties of vector rotators of rigid body dynamics with coupled rotations around axes without intersection as well as vector analysis of nonlinear oscillations of gyro-rotors. 


\subsection{Vectors of body mass moments}

Here, we define three kinetic vectors fixed to a certain point and axis passing through the given rigid body point (see Figure 3). The rotation axis of a body is passing through a revolute joint that connects two bodies in the system and it is oriented by the unit vector $n_{i}$ where subscript $\mathrm{i}$ is the same as the subscript of the corresponding body.

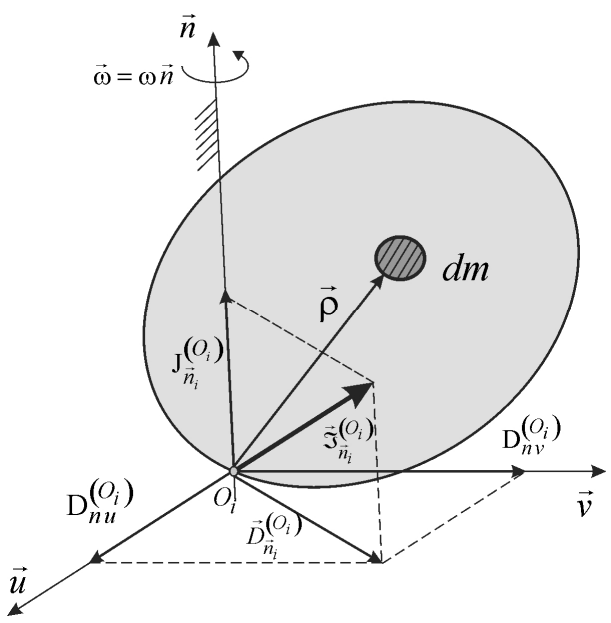

Figure 3. Vectors of body mass moments for the single body rotation around the fixed axes

We give a definition of the vector $\vec{M}_{\vec{n}_{i}}^{\left(O_{i}\right)}$ of the body mass at the point $O_{i}$ for the axis oriented by the unit vector $\vec{n}_{i}$ [8-9]:

$$
\vec{M}_{\vec{n}_{i}}^{\left(O_{i}\right)}:=\iiint_{V} \vec{n} d m=\mathbf{M} \vec{n}, \quad d m=\sigma d V
$$

Then we define vector $\vec{S}_{\vec{n}_{i}}^{\left(O_{i}\right)}$ of the body mass static (linear) moment at the point $O_{i}$ for the axis oriented by the unit vector $\vec{n}_{i}$ in the form [8-9]:

$$
\vec{S}_{\vec{n}_{i}}^{\left(O_{i}\right)}:=\iiint_{V}[\vec{n}, \vec{\rho}] d m=\left[\vec{n}, \vec{\rho}_{c}\right] \mathbf{M}, \quad d m=\sigma d V
$$

The third vector $\overrightarrow{\mathfrak{J}}_{\vec{n}_{i}}^{\left(O_{i}\right)}$ of the body mass inertia at the point $O_{i}$ for the axis oriented by the unit vector $\vec{n}_{i}$ is defined as [8-9]:

$$
\overrightarrow{\mathfrak{J}}_{\vec{n}_{i}}^{\left(O_{i}\right)}:=\iiint_{V}[\vec{\rho},[\vec{n}, \vec{\rho}]] d m n, \quad d m=\sigma d V
$$

where $\vec{\rho}$ is the position vector of the elementary body mass and $\vec{\rho}_{c}$ is the position vector of the centre of body mass with respect to the pole $O_{i}$. The body mass inertia moment vector $\overrightarrow{\mathfrak{J}}_{\vec{n}_{i}}^{\left(O_{i}\right)}$ can be decomposed into three components: the component $\mathrm{J}_{\vec{n}_{i}}^{\left(O_{i}\right)}$ which is co-linear with the axis and two other components $\mathrm{D}_{n u}^{\left(O_{i}\right)}$ and $\mathrm{D}_{n v}^{\left(O_{i}\right)}$ in the directions $\vec{u}$ and $\vec{v}$ which are normal to the orientation axis $\vec{n}$. The co-linear component represents the axial moment of the body mass inertia and the other two components represent the deviational moments:

$$
\overrightarrow{\mathfrak{J}}_{\vec{n}_{i}}^{\left(O_{i}\right)}=\mathrm{J}_{\vec{n}_{i}}^{\left(O_{i}\right)} \vec{n}+\mathrm{D}_{n u}^{\left(O_{i}\right)} \vec{u}+\mathrm{D}_{n v}^{\left(O_{i}\right)} \vec{v}
$$

where $\mathbf{J}_{\vec{n}_{i}}^{\left(O_{i}\right)}$ is:

$$
\mathrm{J}_{\vec{n}_{i}}^{\left(O_{i}\right)}=\iiint_{V}[\vec{n}, \vec{\rho}]^{2} d m n=\left(\overrightarrow{\mathfrak{J}}_{\vec{n}_{i}}^{\left(O_{i}\right)}, \vec{n}\right)
$$

The rigid body deviation moment vector at the point for the axis oriented by the unit vector has the following form:

$$
\overrightarrow{\mathrm{D}}_{\vec{n}_{i}}^{\left(O_{i}\right)}=\iiint_{V}[\vec{n},[[\vec{\rho},[\vec{n}, \vec{\rho}]], \vec{n}]] d m=\left[\vec{n},\left[\overrightarrow{\mathfrak{J}}_{\vec{n}_{i}}^{\left(O_{i}\right)}, \vec{n}\right]\right]
$$

\subsection{Model of a three-like rigid body system}

In this section we observe the same system as in the previous one but with different notation for the body vectors (see Figure 4). Allow us first to consider rotation of the rigid body $\left(V_{1}\right)$ around fixed axis oriented with the unit vector $\vec{n}_{1}$ with angular velocity $\vec{\omega}_{1}=\omega_{1} \vec{n}_{1}$. In a similar way angular velocities around moving axes oriented with unit vectors $\vec{n}_{2}$ and $\vec{n}_{3}$ are $\vec{\omega}_{2}=\omega_{2} \vec{n}_{2}$ and $\vec{\omega}_{3}=\omega_{3} \vec{n}_{3}$, respectively. Joints, whose positions with respect to the first joint $O_{1}$ are determined with $\vec{r}_{12}$ and $\vec{r}_{23}$ vectors, are denoted with $\mathrm{O}_{2}$ and $\mathrm{O}_{3}$ and they coincide with points which are the shortest orthogonal distance between axes oriented with unit vectors $\vec{n}_{1}, \vec{n}_{2}$ and $\vec{n}_{3}$. Axes of rotation are without intersections.

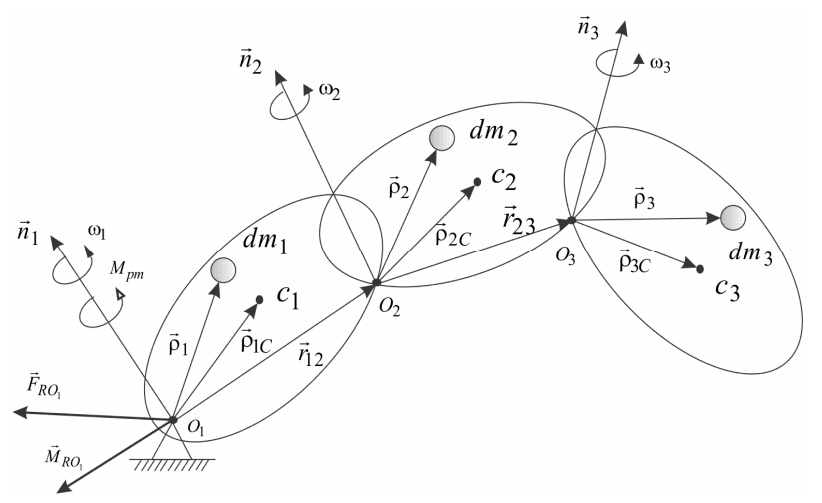

Figure 4. Three-like rigid multibody system with vectors used in a vector method

Here $\vec{\rho}_{i c}$ and $\vec{\rho}_{i}, i=1,2,3$ are position vectors of the body mass centre and elementary body mass with respect to the joint of $i$-th body, respectively. Position vectors of elementary mass with origin in joint $O_{1}$ are introduced with vectors $\vec{r}_{i}, i=1,2,3$ in the form:

$$
\vec{r}_{1}=\vec{\rho}_{1}, \vec{r}_{2}=\vec{r}_{12}+\vec{\rho}_{2}, \vec{r}_{2}=\vec{r}_{12}+\vec{r}_{23}+\vec{\rho}_{3}
$$

and velocities of mass particles $\vec{v}_{i}, i=1,2,3$ in the form

$$
\begin{aligned}
& \vec{v}_{1}=\left[\vec{\omega}_{1}, \vec{\rho}_{1}\right], \vec{v}_{2}=\left[\vec{\omega}_{1}, \vec{r}_{12}\right]+\left[\vec{\omega}_{1}+\vec{\omega}_{2}, \vec{\rho}_{2}\right], \\
& \vec{v}_{3}=\left[\vec{\omega}_{1}, \vec{r}_{12}\right]+\left[\vec{\omega}_{1}+\vec{\omega}_{2}, \vec{r}_{23}\right]+\left[\vec{\omega}_{1}+\vec{\omega}_{2}+\vec{\omega}_{3}, \vec{\rho}_{3}\right]
\end{aligned}
$$




\subsection{Vector equations of linear momentum and angular momentum}

Using the basic definition of linear momentum and angular momentum and basic equations for velocity of mass particles, the following vector equations can be written:

1) For linear momentum in the form

$$
\begin{aligned}
\overrightarrow{\mathrm{K}} & =\iiint_{V_{1}} \vec{v}_{1} d m_{1}+\iiint_{V_{2}} \vec{v}_{2} d m_{2}+\iiint_{V_{3}} \vec{v}_{3} d m_{3} \\
& =\omega_{1} \overrightarrow{\mathrm{S}}_{\left(\vec{n}_{1}\right)}^{\left(O_{1}\right)(1)}+\omega_{1}\left[\vec{n}_{1}, \vec{r}_{12}\right]\left(M_{2}+M_{3}\right)+\omega_{1} \overrightarrow{\mathrm{S}}_{\left(\vec{n}_{1}\right)}^{\left(O_{2}\right)(2)} \\
& +\omega_{2} \overrightarrow{\mathrm{S}}_{\left(O_{2}\right)}^{\left(O_{2}\right)(2)}+\omega_{1}\left[\vec{n}_{1}, \vec{r}_{23}\right] M_{3}+\omega_{2}\left[\vec{n}_{2}, \vec{r}_{23}\right] M_{3} \\
& +\omega_{1} \overrightarrow{\mathrm{S}}_{\left(\vec{n}_{1}\right)}^{\left(O_{3}\right)(3)}+\omega_{1} \overrightarrow{\mathrm{S}}_{\left(\vec{n}_{2}\right)}^{\left(O_{3}\right)(3)}+\omega_{1} \overrightarrow{\mathrm{S}}_{\left(\vec{n}_{2}\right)}^{\left(O_{3}\right)(3)},
\end{aligned}
$$

where $\vec{S}_{\vec{n}_{i}}^{\left(O_{i}\right)}, i=1,2,3$ are body linear mass moments for corresponding points and axes which are written as:

$$
\begin{aligned}
& \vec{S}_{\left(\vec{n}_{1}\right)}^{\left(O_{1}\right)(1)}=\iiint_{V}\left[\vec{n}_{1}, \vec{\rho}_{1}\right] d m_{1}=\left[\vec{n}_{1}, \vec{\rho}_{1 \mathrm{C}}\right] M_{1}, \\
& \vec{S}_{\left(\vec{n}_{1}\right)}^{\left(O_{2}\right)(2)}=\iiint_{V}\left[\vec{n}_{1}, \vec{\rho}_{2}\right] d m_{2}, \\
& \vec{S}_{\left(\vec{n}_{1}\right)}^{\left(O_{2}\right)(2)}=\iiint_{V}\left[\vec{n}_{2}, \vec{\rho}_{2}\right] d m_{2}, \\
& \vec{S}_{\left(\vec{n}_{1}\right)}^{\left(O_{3}\right)(3)}=\iiint \int_{V}\left[\vec{n}_{1}, \rho_{3}\right] d m_{3}, \\
& \vec{S}_{\left(\vec{n}_{2}\right)}^{\left(O_{3}\right)(3)}=\iiint_{V}\left[\vec{n}_{2}, \vec{\rho}_{3}\right] d m_{3}, \\
& \vec{S}_{\left(\vec{n}_{3}\right)}^{\left(O_{3}\right)(3)}=\iiint_{V}\left[\vec{n}_{3}, \vec{\rho}_{3}\right] d m_{3}
\end{aligned}
$$

2) For angular momentum the expression is in the form

$$
\begin{aligned}
& \vec{L}=\iiint_{V}\left[\vec{r}_{1}, \vec{v}_{1}\right] d m_{1}+\iiint_{V}\left[\vec{r}_{2}, \vec{v}_{2}\right] d m_{2}+\iiint_{V}\left[\vec{r}_{3}, \vec{v}_{3}\right] d m_{3} \\
& =\omega_{1} \overrightarrow{\mathfrak{I}}_{\left(\vec{n}_{1}\right)}^{\left(O_{1}\right)(1)}+\omega_{1}\left[\vec{r}_{12},\left[\vec{n}_{1}, \vec{r}_{12}\right]\right] M_{2}+\omega_{1}\left[\vec{r}_{12}, \vec{S}_{\left(\vec{n}_{1}\right)}^{\left(O_{2}\right)(2)}\right] \\
& +\omega_{2}\left[\vec{r}_{12}, \vec{S}_{\left(\vec{n}_{2}\right)}^{\left(O_{2}\right)(2)}\right]+\omega_{1}\left[\vec{\rho}_{2 C},\left[\vec{n}_{1}, \vec{r}_{12}\right]\right] M_{2} \\
& +\omega_{1} \overrightarrow{\mathfrak{I}}_{\left(\vec{n}_{1}\right)}^{\left(O_{2}\right)(2)}+\omega_{2} \overrightarrow{\mathfrak{I}}_{\left(\vec{n}_{2}\right)}^{\left(O_{2}\right)(2)} \\
& +\omega_{1}\left[\vec{r}_{12},\left[\vec{n}_{1}, \vec{r}_{12}\right]\right] M_{3}+\omega_{1}\left[\vec{r}_{12},\left[\vec{n}_{1}, \vec{r}_{23}\right]\right] M_{3} \\
& +\omega_{2}\left[\vec{r}_{12},\left[\vec{n}_{2}, \vec{r}_{23}\right]\right] M_{3}+\omega_{1}\left[\vec{r}_{12}, \vec{S}_{\left(\vec{n}_{1}\right)}^{\left(O_{3}\right)(3)}\right] \\
& +\omega_{2}\left[\vec{r}_{12}, \vec{S}_{\left(\vec{n}_{2}\right)}^{\left(O_{3}\right)(3)}\right]+\omega_{3}\left[\vec{r}_{12}, \vec{S}_{\left(\vec{n}_{3}\right)}^{\left(O_{3}\right)(3)}\right] \\
& +\omega_{1}\left[\vec{r}_{23},\left[\vec{n}_{1}, \vec{r}_{12}\right]\right] M_{3}+\omega_{1}\left[\vec{r}_{23},\left[\vec{n}_{1}, \vec{r}_{23}\right]\right] M_{3} \\
& +\omega_{2}\left[\vec{r}_{23},\left[\vec{n}_{2}, \vec{r}_{23}\right]\right] M_{3}+\omega_{1}\left[\vec{r}_{23}, \vec{S}_{\left(\vec{n}_{1}\right)}^{\left(O_{3}\right)(3)}\right] \\
& +\omega_{2}\left[\vec{r}_{23}, \vec{S}_{\left(\vec{n}_{2}\right)}^{\left(O_{3}\right)(3)}\right]+\omega_{3}\left[\vec{r}_{23}, \vec{S}_{\left(\vec{n}_{3}\right)}^{\left(O_{3}\right)(3)}\right] \\
& +\omega_{1}\left[\vec{\rho}_{3 C},\left[\vec{n}_{1}, \vec{r}_{12}\right]\right] M_{3}+\omega_{1}\left[\vec{\rho}_{3 C},\left[\vec{n}_{1}, \vec{r}_{23}\right]\right] M_{3}
\end{aligned}
$$

$+\omega_{1}\left[\vec{\rho}_{3 C},\left[\vec{n}_{1}, \vec{r}_{23}\right]\right] M_{3}+\omega_{2}\left[\vec{\rho}_{3 C},\left[\vec{n}_{2}, \vec{r}_{23}\right]\right] M_{3}$

$+\omega_{1} \overrightarrow{\mathfrak{I}}_{\left(\vec{n}_{1}\right)}^{\left(O_{3}\right)(3)}+\omega_{2} \overrightarrow{\mathfrak{J}}_{\left(\vec{n}_{2}\right)}^{\left(O_{3}\right)(3)}+\omega_{3} \overrightarrow{\mathfrak{I}}_{\left(\vec{n}_{3}\right)}^{\left(O_{3}\right)(3)}$,

where $\overrightarrow{\mathfrak{I}}_{\left(\vec{n}_{i}\right)}^{\left(O_{j}\right)(j)}, i, j=1,2,3$ are corresponding rigid body mass inertia moment vectors for axes oriented by directions of component rotations through the joints $O_{i}, i=1$, 2, 3 on self-rotating axes written in the following form:

$$
\begin{aligned}
& \overrightarrow{\mathfrak{I}}_{\left(\vec{n}_{1}\right)}^{\left(O_{1}\right)(1)}=\iiint_{V}\left[\vec{\rho}_{1},\left[\vec{n}_{1}, \vec{\rho}_{1}\right]\right] d m_{1}, \\
& \overrightarrow{\mathfrak{I}}_{\left(\vec{n}_{1}\right)}^{\left(O_{2}\right)(2)}=\iiint_{V 2}\left[\vec{\rho}_{2},\left[\vec{n}_{1}, \vec{\rho}_{2}\right]\right] d m_{2}, \\
& \overrightarrow{\mathfrak{I}}_{\left(\vec{n}_{2}\right)}^{\left(O_{2}\right)(2)}=\iiint_{V 2}\left[\vec{\rho}_{2},\left[\vec{n}_{2}, \vec{\rho}_{2}\right]\right] d m_{2}, \\
& \overrightarrow{\mathfrak{I}}_{\left(\vec{n}_{1}\right)}^{\left(O_{3}\right)(3)}=\iiint_{V 3}\left[\vec{\rho}_{3},\left[\vec{n}_{1}, \vec{\rho}_{3}\right]\right] d m_{3}, \\
& \overrightarrow{\mathfrak{I}}_{\left(\vec{n}_{2}\right)}^{\left(O_{3}\right)(3)}=\iiint_{V 3}\left[\vec{\rho}_{3},\left[\vec{n}_{2}, \vec{\rho}_{3}\right]\right] d m_{3}, \\
& \overrightarrow{\mathfrak{I}}_{\left(\vec{n}_{3}\right)}^{\left(O_{3}\right)(3)}=\iiint_{V 3}\left[\vec{\rho}_{3},\left[\vec{n}_{3}, \vec{\rho}_{3}\right]\right] d m_{3}
\end{aligned}
$$

\subsection{Derivatives of linear momentum and angular momentum}

We take into account the equation for linear momentum (48) and find derivative of parts, after rearranging the terms we obtain the following vector expression

$$
\begin{aligned}
& \frac{d \vec{K}}{d t}=\dot{\omega}_{1} \vec{S}_{\left(\vec{n}_{1}\right)}^{\left(O_{1}\right)(1)}+\omega_{1}^{2}\left[\vec{n}_{1}, \vec{S}_{\left(\vec{n}_{1}\right)}^{\left(O_{1}\right)(1)}\right]+\dot{\omega}_{1}\left[\vec{n}_{1}, \vec{r}_{12}\right] M_{2} \\
& +\omega_{1}^{2}\left[\vec{n}_{1},\left[\vec{n}_{1}, \vec{r}_{12}\right]\right] M_{2}+\dot{\omega}_{1} \vec{S}_{\left(\vec{n}_{1}\right)}^{\left(O_{2}\right)(2)}+\omega_{1}^{2}\left[\vec{n}_{1}, \vec{S}_{\left(\vec{n}_{1}\right)}^{\left(O_{2}\right)(2)}\right] \\
& +\dot{\omega}_{2} \vec{S}_{\left(\vec{n}_{2}\right)}^{\left(O_{2}\right)(2)}+\omega_{2}^{2}\left[\vec{n}_{2}, \vec{S}_{\left(\vec{n}_{1}\right)}^{\left(O_{2}\right)(2)}\right]+2 \omega_{1} \omega_{2}\left[\vec{n}_{1}, \vec{S}_{\left(\overrightarrow{n_{2}}\right)}^{\left(O_{2}\right)(2)}\right] \\
& +\dot{\omega}_{1}\left[\vec{n}_{1}, \vec{r}_{12}\right] M_{3}+\omega_{1}^{2}\left[\vec{n}_{1},\left[\vec{n}_{1}, \vec{r}_{12}\right]\right] M_{3}+\dot{\omega}_{1}\left[\vec{n}_{1}, \vec{r}_{23}\right] M_{3} \\
& +\omega_{1}^{2}\left[\vec{n}_{1},\left[\vec{n}_{1}, \vec{r}_{23}\right]\right] M_{3}+\dot{\omega}_{2}\left[\vec{n}_{2}, \vec{r}_{23}\right] M_{3} \\
& +2 \omega_{1} \omega_{3}\left[\vec{n}_{1}, \vec{S}_{\left(\vec{n}_{3}\right)}^{\left(O_{3}\right)(3)}\right]+2 \omega_{2} \omega_{3}\left[\vec{n}_{2}, \vec{S}_{\left(\vec{n}_{3}\right)}^{\left(O_{3}\right)(3)}\right] \\
& +2 \omega_{1} \omega_{2}\left[\vec{n}_{1},\left[\vec{n}_{2}, \vec{r}_{23}\right]\right] M_{3}+\omega_{2}^{2}\left[\vec{n}_{2},\left[\vec{n}_{2}, \vec{r}_{23}\right]\right] M_{3} \\
& +\dot{\omega}_{1}\left[\vec{n}_{1}, \vec{\rho}_{3}\right] M_{3}+\omega_{1}^{2}\left[\vec{n}_{1}, \vec{S}_{\left(\vec{n}_{1}\right)}^{\left(O_{3}\right)(3)}\right]+2 \omega_{1} \omega_{2}\left[\vec{n}_{1}, \vec{S}_{\left(\vec{n}_{2}\right)}^{\left(O_{3}\right)(3)}\right] \\
& +\dot{\omega}_{2} \vec{S}_{\left(\vec{n}_{2}\right)}^{\left(O_{3}\right)(3)}+\omega_{2}^{2}\left[\vec{n}_{2}, \vec{S}_{\left(\vec{n}_{2}\right)}^{\left(O_{3}\right)(3)}\right] \\
& +\dot{\omega}_{3} \vec{S}_{\left(\vec{n}_{3}\right)}^{\left(O_{3}\right)(3)}+\omega_{3}^{2}\left[\vec{n}_{3}, \vec{S}_{\left(\vec{n}_{3}\right)}^{\left(O_{3}\right)(3)}\right] .
\end{aligned}
$$

By analysing the structure of linear momentum derivative terms, one can see that it is possible to introduce pure kinematic vectors, so called vector rotators [8-12], depending on the component angular velocities and component angular accelerations of the component coupled rotations which are given as follows: 


$$
\begin{aligned}
& \vec{\Re}_{30}=\dot{\omega}_{1} \frac{\left[\vec{n}_{1}, \vec{r}_{12}\right]}{\left|\left[\vec{n}_{1}, \vec{r}_{12}\right]\right|}+\omega_{1}^{2}\left[\vec{n}_{1}, \frac{\left[\vec{n}_{1}, \vec{r}_{12}\right]}{\left|\left[\vec{n}_{1}, \vec{r}_{12}\right]\right|}\right], \\
& \vec{\Re}_{3}=\dot{\omega}_{1} \frac{\left[\vec{n}_{1}, \vec{r}_{23}\right]}{\left[\vec{n}_{1}, \vec{r}_{23}\right]}+\omega_{1}^{2}\left[\vec{n}_{1}, \frac{\left[\vec{n}_{1}, \vec{r}_{23}\right]}{\left[\vec{n}_{1}, \vec{r}_{23}\right]}\right], \\
& \vec{\Re}_{31}=\dot{\omega}_{2} \frac{\left[\vec{n}_{2}, \vec{r}_{23}\right]}{\left[\vec{n}_{2}, \vec{r}_{23}\right]}+\omega_{2}^{2}\left[\vec{n}_{2}, \frac{\left[\vec{n}_{2}, \vec{r}_{23}\right]}{\left[\vec{n}_{2}, \vec{r}_{23}\right]}\right] \text {, } \\
& \vec{\Re}_{32}=2 \omega_{1} \omega_{2}\left[\vec{n}_{1}, \frac{\left[\vec{n}_{2}, \vec{r}_{23}\right]}{\left[\vec{n}_{2}, \vec{r}_{23}\right]}\right] \text {, }
\end{aligned}
$$

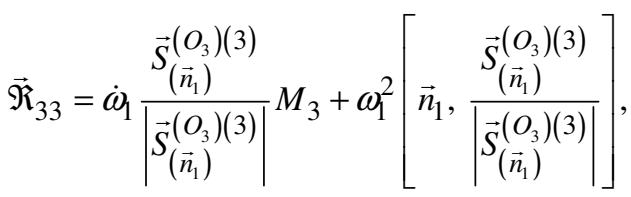

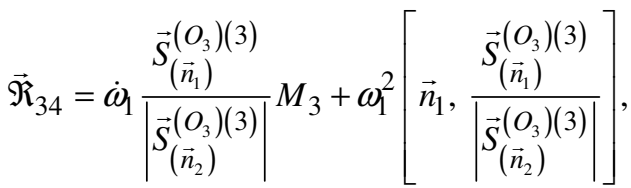

$$
\begin{aligned}
& \vec{\Re}_{35}=2 \omega_{1} \omega_{2}\left[\vec{n}_{1}, \frac{\vec{S}_{\left(\vec{n}_{2}\right)}^{\left(O_{3}\right)(3)}}{\left|\vec{S}_{\left(\vec{n}_{2}\right)}^{\left(O_{3}\right)(3)}\right|}\right] \text {, } \\
& \vec{\Re}_{36}=\dot{\omega}_{3} \frac{\vec{S}_{\left(\vec{n}_{3}\right)}^{\left(O_{3}\right)(3)}}{\left|\vec{S}_{\left(\vec{n}_{3}\right)}^{\left(O_{3}\right)(3)}\right|}+\omega_{3}^{2}\left[\vec{n}_{3}, \frac{\vec{S}_{\left(\vec{n}_{3}\right)}^{\left(O_{3}\right)(3)}}{\left|\vec{S}_{\left(\vec{n}_{3}\right)}^{\left(O_{3}\right)(3)}\right|}\right], \\
& \vec{\Re}_{37}=2 \omega_{1} \omega_{3}\left[\vec{n}_{1}, \frac{\vec{S}_{\left(\vec{n}_{3}\right)}^{\left(O_{3}\right)(3)}}{\mid \vec{S}_{\left(\vec{n}_{3}\right)}^{\left(O_{3}\right)(3) \mid}}\right] \text {, } \\
& \vec{\Re}_{38}=2 \omega_{2} \omega_{3}\left[\vec{n}_{2}, \frac{\vec{S}_{\left(\vec{n}_{3}\right)}^{\left(O_{3}\right)(3)}}{\left|\vec{S}_{\left(\vec{n}_{3}\right)}^{\left(O_{3}\right)(3)}\right|}\right]
\end{aligned}
$$

Then, we can express derivatives of the linear momentum in the following form:

$$
\begin{aligned}
& \frac{d \vec{K}}{d t}=\vec{\Re}_{1}\left|\vec{S}_{\left(\vec{n}_{1}\right)}^{\left(O_{1}\right)(1)}\right|+\vec{\Re}_{20}\left|\left[\vec{n}_{1}, \overrightarrow{r_{12}}\right]\right| M_{2}+\vec{\Re}_{2}\left|\vec{S}_{\left(\vec{n}_{1}\right)}^{\left(O_{2}\right)(2)}\right| \\
& +\vec{\Re}_{21}\left|\vec{S}_{\left(\vec{n}_{2}\right)}^{\left(O_{2}\right)(2)}\right|+\vec{\Re}_{22}\left|\vec{S}_{\left(\vec{n}_{2}\right)}^{\left(O_{2}\right)(2)}\right| \\
& +\vec{\Re}_{30}\left[\vec{n}_{1}, \vec{r}_{12}\right] M_{3}+\vec{\Re}_{3} \mid\left[\vec{n}_{1}, \vec{r}_{23}\right] M_{3} \\
& +\vec{\Re}_{31}\left[\vec{n}_{2}, \vec{r}_{23}\right] M_{3}+\vec{\Re}_{32}\left[\vec{n}_{2}, \vec{r}_{23}\right] M_{3} \\
& +\vec{\Re}_{33}\left|\vec{S}_{\left(\vec{n}_{1}\right)}^{\left(O_{3}\right)(3)}\right|+\vec{\Re}_{34}\left|\vec{S}_{\left(\vec{n}_{2}\right)}^{\left(O_{3}\right)(3)}\right| \\
& +\vec{\Re}_{35}\left|\vec{S}_{\left(\vec{n}_{2}\right)}^{\left(O_{3}\right)(3)}\right|+\vec{\Re}_{36}\left|\vec{S}_{\left(\vec{n}_{3}\right)}^{\left(O_{3}\right)(3)}\right| \\
& +\vec{\Re}_{37}\left|\vec{S}_{\left(\vec{n}_{3}\right)}^{\left(O_{3}\right)(3)}\right|+\vec{\Re}_{38}\left|\vec{S}_{\left(\vec{n}_{3}\right)}^{\left(O_{3}\right)(3)}\right| \\
& +\vec{\Re}_{31} \mid\left[\vec{n}_{2}, \vec{r}_{23}\right] M_{3}+\vec{\Re}_{32}\left[\left[\vec{n}_{2}, \vec{r}_{23}\right] M_{3}\right.
\end{aligned}
$$

$$
\begin{aligned}
& +\vec{\Re}_{33}\left|\vec{S}_{\left(\vec{n}_{1}\right)}^{\left(O_{3}\right)(3)}\right|+\vec{\Re}_{34}\left|\vec{S}_{\left(\vec{n}_{2}\right)}^{\left(O_{3}\right)(3)}\right| \\
& +\vec{\Re}_{35}\left|\vec{S}_{\left(\vec{n}_{2}\right)}^{\left(O_{3}\right)(3)}\right|+\vec{\Re}_{36}\left|\vec{S}_{\left(\vec{n}_{3}\right)}^{\left(O_{3}\right)(3)}\right| \\
& +\vec{\Re}_{37}\left|\vec{S}_{\left(\vec{n}_{3}\right)}^{\left(O_{3}\right)(3)}\right|+\vec{\Re}_{38}\left|\vec{S}_{\left(\vec{n}_{3}\right)}^{\left(O_{3}\right)(3)}\right|
\end{aligned}
$$

In the same manner, we derive the vector expression for angular momentum and due to the large equations we express linear momentum derivatives by using the vector rotators and obtain the form:

$\frac{d \vec{L}}{d t}=\vec{n}_{1} \dot{\omega}_{1}\left(\vec{n}_{1}, \overrightarrow{\mathfrak{I}}_{\left(\vec{n}_{1}\right)}^{\left(O_{1}\right)(1)}\right)+\vec{\Re}_{1}^{*}\left|\vec{D}_{\left(\vec{n}_{1}\right)}^{\left(O_{1}\right)(1)}\right|$

$+\left|\left[\vec{n}_{1}, \vec{r}_{12}\right]\right|\left[\vec{r}_{12}, \vec{\Re}_{20}\right] M_{2}+\left[\vec{n}_{1}, \vec{r}_{12}\right] \mid\left[\vec{\rho}_{2 C}, \vec{\Re}_{20}\right] M_{2}$

$+\left|\vec{S}_{\left(\vec{n}_{1}\right)}^{\left(O_{2}\right)(2)}\right|\left[\vec{r}_{12}, \vec{\Re}_{2}\right]$

$+\left|\vec{S}_{\left(\vec{n}_{2}\right)}^{\left(O_{2}\right)(2)}\right|\left[\vec{r}_{12}, \vec{\Re}_{22}\right]$

$+\left|\left[\vec{n}_{1}, \vec{S}_{\left(\vec{n}_{2}\right)}^{\left(O_{2}\right)(2)}\right]\right|\left[\vec{r}_{12}, \vec{\Re}_{21}\right]$

$+\vec{n}_{1} \dot{\omega}_{1}\left(\vec{n}_{1}, \overrightarrow{\mathfrak{J}}_{\left(\overrightarrow{n_{1}}\right)}^{\left(O_{2}\right)(2)}\right)+\vec{\Re}_{2}^{*}\left|\vec{D}_{\left(\vec{n}_{1}\right)}^{\left(O_{2}\right)(2)}\right|$

$+\overrightarrow{n_{2}} \dot{\omega}_{2}\left(\vec{n}_{2}, \vec{\Im}_{\left(\frac{n_{2}}{n_{2}}\right)}^{\left(O_{2}\right)(2)}\right)+\vec{\Re}_{22}^{*}\left|\vec{D}_{\left(\vec{n}_{2}\right)}^{\left(O_{2}\right)(2)}\right|+$

$+\omega_{1} \omega_{2}\left\{\left[\vec{n}_{1}, \overrightarrow{\mathfrak{J}}_{\left(\vec{n}_{2}\right)}^{\left(O_{2}\right)(2)}\right]+\left[\vec{n}_{2}, \overrightarrow{\mathfrak{J}}_{\left(\vec{n}_{1}\right)}^{\left(O_{2}\right)(2)}\right]+\mathfrak{I}^{\left(O_{2}\right)(2)}\left[\vec{n}_{1}, \vec{n}_{2}\right]\right\}+$

$+\left|\left[\vec{n}_{1}, \vec{r}_{12}\right]\right|\left\{\left[\vec{r}_{12}, \vec{\Re}_{30}\right]\right\} M_{3}$

$+\left[\left[\vec{n}_{1}, \vec{r}_{23}\right]\left\{\left[\vec{r}_{12}, \vec{\Re}_{3}\right]\right\} M_{3}\right.$

$+\left[\left[\vec{n}_{2}, \vec{r}_{23}\right]\left\{\left[\vec{r}_{12}, \vec{\Re}_{31}\right]\right\} M_{3}+\left[\vec{n}_{2}, \vec{r}_{12}\right] \mid\left[\vec{r}_{12}, \vec{\Re}_{32}\right] M_{3}\right.$

$+\left|\vec{S}_{\left(\vec{n}_{1}\right)}^{\left(O_{3}\right)(3)}\right|\left[\vec{r}_{12}, \vec{\Re}_{33}\right]+\left|\vec{S}_{\left(\vec{n}_{2}\right)}^{\left(O_{3}\right)(3)}\right| 2 \omega_{1} \omega_{2}\left[\vec{r}_{12}, \vec{\Re}_{35}\right]$

$+\left|\vec{S}_{\left(\vec{n}_{2}\right)}^{\left(O_{3}\right)(3)}\right|\left[\vec{r}_{12}, \vec{\Re}_{34}\right]+\left|\vec{S}_{\left(\vec{n}_{3}\right)}^{\left(O_{3}\right)(3)}\right|\left[\vec{r}_{12}, \vec{\Re}_{37}\right]$

$+\left|\vec{S}_{\left(\vec{n}_{3}\right)}^{\left(O_{3}\right)(3)}\right|\left[\vec{r}_{12}, \vec{\Re}_{36}\right]+\left|\vec{S}_{\left(\vec{n}_{3}\right)}^{\left(O_{3}\right)(3)}\right|\left[\vec{r}_{12}, \vec{\Re}_{38}\right]$

$+\left|\left[\vec{n}_{1}, \vec{r}_{12}\right]\right|\left\{\left[\vec{r}_{23}, \vec{\Re}_{30}\right]\right\} M_{3}$

$+\left[\vec{n}_{1}, \vec{r}_{23}\right]\left\{\left[\vec{r}_{23}, \vec{\Re}_{3}\right]\right\} M_{3}+\left[\vec{n}_{2}, \vec{r}_{23}\right]\left[\vec{r}_{23}, \vec{\Re}_{32}\right] M_{3}$

$+\left[\vec{n}_{2}, \vec{r}_{23}\right]\left\{\left[\vec{r}_{23}, \vec{\Re}_{31}\right]\right\} M_{3}$

$+\left|\vec{S}_{\left(\vec{n}_{1}\right)}^{\left(O_{3}\right)(3)}\right|\left\{\left[\vec{r}_{23}, \vec{\Re}_{33}\right]\right\} M_{3}+\left|\vec{S}_{\left(\vec{n}_{2}\right)}^{\left(O_{3}\right)(3)}\right|\left[\vec{r}_{23}, \vec{\Re}_{35}\right]$

$+\left|\vec{S}_{\left(\vec{n}_{2}\right)}^{\left(O_{3}\right)(3)}\right|\left\{\left[\vec{r}_{23}, \vec{\Re}_{34}\right]\right\} M_{3}+\left|\vec{S}_{\left(\vec{n}_{3}\right)}^{\left(O_{3}\right)(3)}\right|\left[\vec{r}_{23}, \vec{\Re}_{37}\right]$

$+\left|\vec{S}_{\left(\vec{n}_{3}\right)}^{\left(O_{3}\right)(3)}\right|\left\{\left[\vec{r}_{23}, \vec{\Re}_{36}\right]\right\} M_{3}+\left|\vec{S}_{\left(\vec{n}_{3}\right)}^{\left(O_{3}\right)(3)}\right|\left[\vec{r}_{23}, \vec{\Re}_{38}\right]$

$+\left|\left[\vec{n}_{1}, \vec{r}_{12}\right]\right|\left\{\left[\vec{\rho}_{3 C}, \vec{\Re}_{30}\right]\right\} M_{3}$

$+\left[\vec{n}_{1}, \vec{r}_{23}\right]\left\{\left[\vec{\rho}_{3 C}, \vec{\Re}_{3}\right]\right\} M_{3}$ 


$$
\begin{aligned}
& +\left[\vec{n}_{2}, \vec{r}_{23}\right]\left\{\left[\vec{\rho}_{3 C}, \vec{\Re}_{31}\right]\right\} M_{3}+\left[\left[\vec{n}_{2}, \vec{r}_{23}\right]\left[\vec{\rho}_{3 C}, \vec{\Re}_{32}\right] M_{3}\right. \\
& +\vec{n}_{1} \dot{\omega}_{1}\left(\vec{n}_{1}, \overrightarrow{\mathfrak{J}}_{\left(\vec{n}_{1}\right)}^{\left(O_{3}\right)(3)}\right)+\overrightarrow{\mathfrak{R}}_{30}^{*}\left|\vec{D}_{\left(\vec{n}_{1}\right)}^{\left(O_{3}\right)(3)}\right| \\
& +\vec{n}_{2} \dot{\omega}_{2}\left(\vec{n}_{2}, \overrightarrow{\mathfrak{J}}_{\left(\vec{n}_{2}\right)}^{\left(O_{3}\right)(3)}\right)+\vec{\Re}_{40}^{*}\left|\vec{D}_{\left(\vec{n}_{2}\right)}^{\left(O_{3}\right)(3)}\right| \\
& +\vec{n}_{3} \dot{\omega}_{3}\left(\vec{n}_{3}, \overrightarrow{\mathfrak{I}}_{\left(\vec{n}_{3}\right)}^{\left(O_{3}\right)(3)}\right)+\overrightarrow{\mathfrak{R}}_{50}^{*}\left|\vec{D}_{\left(\vec{n}_{3}\right)}^{\left(O_{3}\right)(3)}\right| \\
& +\omega_{1} \omega_{2}\left\{\left[\vec{n}_{1}, \overrightarrow{\mathfrak{I}}_{\left(\vec{n}_{2}\right)}^{\left(O_{3}\right)(3)}\right]+\left[\vec{n}_{2}, \overrightarrow{\mathfrak{I}}_{\left(\vec{n}_{1}\right)}^{\left(O_{3}\right)(3)}\right]+\mathfrak{I}^{(3)}\left[\vec{n}_{1}, \vec{n}_{2}\right]\right\} \\
& +\omega_{2} \omega_{3}\left\{\left[\vec{n}_{2}, \overrightarrow{\mathfrak{I}}_{\left(\vec{n}_{3}\right)}^{\left(O_{3}\right)(3)}\right]+\left[\vec{n}_{3}, \overrightarrow{\mathfrak{I}}_{\left(\vec{n}_{2}\right)}^{\left(O_{3}\right)(3)}\right]+\mathfrak{I}^{(3)}\left[\vec{n}_{2}, \vec{n}_{3}\right]\right\}+\omega_{1} \\
& \omega_{3}\left\{\left[\vec{n}_{1}, \overrightarrow{\mathfrak{I}}_{\left(\vec{n}_{3}\right)}^{\left(O_{3}\right)(3)}\right]+\left[\vec{n}_{3}, \overrightarrow{\mathfrak{I}}_{\left(\vec{n}_{1}\right)}^{\left(O_{3}\right)(3)}\right]+\mathfrak{I}^{(3)}\left[\vec{n}_{1}, \vec{n}_{3}\right]\right\},
\end{aligned}
$$

Here we also introduce some new vector rotators which are written in the form

$$
\begin{aligned}
& \vec{\Re}_{1}^{*}=\dot{\omega}_{1} \frac{\vec{D}_{\left(\vec{n}_{1}\right)}^{\left(O_{1}\right)(1)}}{\left|\vec{D}_{\left(\vec{n}_{1}\right)}^{\left(O_{1}\right)(1)}\right|}+\omega_{1}^{2}\left[\vec{n}_{1}, \frac{\vec{D}_{\left(\vec{n}_{1}\right)}^{\left(O_{1}\right)(1)}}{\left|\vec{D}_{\left(\vec{n}_{1}\right)}^{\left(O_{1}\right)(1)}\right|}\right], \\
& \vec{\Re}_{30}^{*}=\dot{\omega}_{1} \frac{\vec{D}_{\left(\vec{n}_{1}\right)}^{\left(O_{3}\right)(3)}}{\left|\vec{D}_{\left(\vec{n}_{1}\right)}^{\left(O_{3}\right)(3)}\right|}+\omega_{1}^{2}\left[\vec{n}_{1}, \frac{\vec{D}_{\left(\vec{n}_{1}\right)}^{\left(O_{3}\right)(3)}}{\left|\vec{D}_{\left(\vec{n}_{1}\right)}^{\left(O_{3}\right)(3)}\right|}\right] \text {, } \\
& \vec{\Re}_{2}^{*}=\dot{\omega}_{1} \frac{\vec{D}_{\left(\overrightarrow{n_{1}}\right)}^{\left(O_{2}\right)(2)}}{\left|\vec{D}_{\left(\overrightarrow{n_{1}}\right)}^{\left(O_{2}\right)(2)}\right|}+\omega_{1}^{2}\left[\overrightarrow{n_{1}}, \frac{\vec{D}_{\left(\overrightarrow{n_{1}}\right)}^{\left(O_{2}\right)(2)}}{\left|\vec{D}_{\left(\overrightarrow{n_{1}}\right)}^{\left(O_{2}\right)(2)}\right|}\right], \\
& \vec{\Re}_{40}^{*}=\dot{\omega}_{2} \frac{\vec{D}_{\left(\vec{n}_{2}\right)}^{\left(O_{3}\right)(3)}}{\left|\vec{D}_{\left(\vec{n}_{2}\right)}^{\left(O_{3}\right)(3)}\right|}+\omega_{2}^{2}\left[\vec{n}_{2}, \frac{\vec{D}_{\left(\vec{n}_{2}\right)}^{\left(O_{3}\right)(3)}}{\left|\vec{D}_{\left(\vec{n}_{2}\right)}^{\left(O_{3}\right)(3)}\right|}\right] \text {, } \\
& \vec{\Re}_{22}^{*}=\dot{\omega}_{2} \frac{\vec{D}_{\left(\vec{n}_{2}\right)}^{\left(O_{2}\right)(2)}}{\left|\vec{D}_{\left(\vec{n}_{2}\right)}^{\left(O_{2}\right)(2)}\right|}+\omega_{2}^{2}\left[\vec{n}_{2}, \frac{\vec{D}_{\left(\vec{n}_{2}\right)}^{\left(O_{2}\right)(2)}}{\left|\vec{D}_{\left(\vec{n}_{2}\right)}^{\left(O_{2}\right)(2)}\right|}\right] \text {, } \\
& \vec{\Re}_{50}^{*}=\dot{\omega}_{3} \frac{\vec{D}_{\left(\vec{n}_{3}\right)}^{\left(O_{3}\right)(3)}}{\left|\vec{D}_{\left(\vec{n}_{3}\right)}^{\left(O_{3}\right)(3)}\right|}+\omega_{3}^{2}\left[\vec{n}_{3}, \frac{\vec{D}_{\left(\vec{n}_{3}\right)}^{\left(O_{3}\right)(3)}}{\left|\vec{D}_{\left(\vec{n}_{3}\right)}^{\left(O_{3}\right)(3)}\right|}\right]
\end{aligned}
$$

\subsection{Determination of joint reactions by using the method based on vectors of body mass moments and vector rotators}

By applying theorems of change of linear momentum and angular momentum we write the following expressions:

$$
\begin{gathered}
\frac{d \vec{K}}{d t}=\vec{F}_{R O_{k}}+\sum_{i=k}^{3} \vec{G}_{i} \\
\frac{d \vec{L}}{d t}=\vec{M}_{R O_{k}}+\sum_{i=k}^{3}\left[\vec{r}_{c i}, \vec{G}_{i}\right]+\vec{M}_{k}
\end{gathered}
$$

where in (56), $\vec{F}_{R O_{k}}$ denotes the resultant joint re-action force in the $k$-th joint and $\vec{G}_{i}$, denotes gravitation forces. In eq. (58) $\vec{M}_{R O_{k}}$ denotes the resultant joint reaction moment in the $k$-th joint, $\left\lfloor\vec{r}_{c i}, \vec{G}_{i}\right\rfloor$, denotes the moments of gravitational forces and a actuator torque $\vec{M}_{k}$ for the revolute joint $O_{k}, k=1,2,3$. From previous two equations it is easy to determine the resultant joint reaction force and moment in the first joint as:

$$
\begin{gathered}
\vec{F}_{R O_{1}}=\frac{d \vec{K}}{d t}-\vec{G}_{1}-\vec{G}_{2}-\vec{G}_{3} \\
\vec{M}_{R O_{1}}=\frac{d \vec{L}}{d t}-\left[\vec{r}_{c 1}, \vec{G}_{1}\right]-\left[\vec{r}_{c 2}, \vec{G}_{2}\right]-\left[\vec{r}_{c 3}, \vec{G}_{3}\right]-\vec{M}_{1}(60)
\end{gathered}
$$

The similar procedure is for determination of the resultant joint reaction forces and moments in other two joints of the three-like rigid body system.

It is known that joint reactions can be split in to the static parts and the kinetic parts, where negative values of kinetic parts are called kinetic pressures. Observing this problem could be of great importance in the systems where the period of change of kinetic pressure overlaps with the period of the eigenfrequency of the joint shaft, when resonance can occur and cause damage of the system. This problem is more frequent in the systems with fast-rotating body parts and it is less frequent in robotic applications. However, we can say that using the vector method described in this section it is easy to obtain kinetic reactions. From (57) and (58) by taking into account (54), (55) and by neglecting the terms with external forces we can obtain kinetic reactions with components in the directions of vector rotators and with magnitudes as products of deviation moments of masses and magnitudes of vector rotators.

\section{CONCLUSION}

In this paper, two different analytic methods for determination of joint reaction forces and moments are presented. The first method based on the Rodrigues approach is applied for determination of the resultant joint reaction forces and moments. Taking into acount that the obtained equations are convenient for a symbolic computation (Mathematica, MatLab, Maple etc.), at this moment it is easy to implement this method in the multibody dynamic problems, especially if there are more than three bodies in the system. The second method based on the vectors of body mass moments and vector rotators for corresponding poles and axes is introduced through the example of three-rigid body system. The expressions for linear and angular momentum are derived and the resultant reaction force and the moment for the fixed joint in the system are determined. In the literature it was shown that this method is convenient to separate joint raction forces into its components ie. to determine kinetic pressures for the corresponding joints. Still, for this method it is needed to develop corresponding algorithms and computer tools so that large analytic expressions could be more easily applied in multibody dynamics. 


\section{ACKNOWLEDGMENT}

This research was sponsored by the research grants of the Serbian Ministry of Education, Science and Techno-logical Development under the numbers OI 174001 and TR 35006.

\section{REFERENCES}

[1] Čović, V., Lukačević, M.: Contribution to the dynamic problems with friction, ZAMM, Vol. 67, No. 4, pp. T67-T70, 1987.

[2] Haug, J.E., Wu, C.S., Yang M.S.: Dynamics of mechanical systems with Coloumb friction, stiction, impact and constraint addition-deletion-I, Mechanism and Machine Theory, Vol. 21, No. 5, pp. 401-406, 1986.

[3] Wu, C.S., Yang, M.S., Haug, J.E.: Dynamics of mechanical systems with Coloumb friction, stiction, impact and constraint addition-deletion-II planar sytem, Mechanism and Machine Theory, Vol. 21, No. 5, pp. 407-4016, 1986.

[4] Wu, C.S., Yang, M.S., Haug, J.E.: Dynamics of mechanical systems with Coloumb friction, stiction, impact and constraint addition-deletion-III spatial systems, Mechanism and Machine Theory, Vol. 21, No. 5, pp. 401-406, 1986.

[5] Šalinić, S.: Determination of joint reaction forces in a symbolic form in rigid multibody systems, Mechanism and Machine Theory, Vol. 46, No. 11, pp. 1796-1810, 2011.

[6] Marković, D.S.: Automated setting of differential equations of motion of rigid body systems in analytic shape for general case with application to technical objects, $\mathrm{PhD}$ thesis, Faculty of Mechanical Engineering, University of Belgrade, Belgrade, 1996.

[7] Čović, V., Lazarević, M.: Mechanics of robots, Faculty of Mechanical engineering, University of Belgrade, 2009.
[8] Hedrih (Stevanović), R.K.: On some interpretations of the rigid bodies kinetic parameters, XVIIIth ICTAM HAIFA, Abstracts, pp. 73-74, 1992.

[9] Hedrih (Stevanovic), R.K.: The vector method of the heavy rotor kinetic parameter analysis and nonlinear dynamics, University of Niš, Niš, 2001.

[10] Hedrih (Stevanovic), R.K.: Vector method based on mass moment vectors and vector rotators applied to rigid-body multi-coupled rotations around no intersecting axes, International Journal of Structural Stability and Dynamics, Vol. 13, No. 7, 2013.

[11] Hedrih (Stevanovic்), R.K., Veljovic, Lj.: Vector rotators of rigid body dynamics with coupled rotations around axes without intersection, Mathematical Problems in Engineering, Article ID 351269, pp. 1-27, 2011.

[12] Veljović, Lj.: Nonlinear oscillations of gyro-rotors, $\mathrm{PhD}$ thesis, Faculty of Mechanical Engineering, University of Niš, Niš, 2011.

\section{ОДРЕБИВАЊЕ РЕАКЦИЈА ЗГЛОБА У СИСТЕМУ ВИШЕ КРУТИХ ТЕЛА, ДВА РАЗЛИЧИТА ПРИСТУПА}

\section{Милан С. Цајић, Михаило П. Лазаревић}

У овом раду су приказане две различите методе за одређивање сила и момената реакција идеалних веза у зглобовима. Разматрани систем више тела има структуру отвореног кинематског ланца. Прва метода се односи на одређивање главних вектора и момената реакција веза у симболичкој форми која се заснива на Родригезовом приступу и погодна је за симболичко рачунање. Друга приказана метода је векторска метода која је базирана на векторима момената маса и векторима ротаторима везаних за пол и усмерену осу. Оба примера су приказана и дискутована на систему три крута тела. 